\title{
Intervenção comportamental para melhorar a precisão do "chute no gol" em jogadores de futebol
}

\author{
Behavioral intervention to improve the accuracy \\ of "kicking the ball into the goal" in soccer players \\ Intervención conductual para mejorar la precisión \\ del "tiro hacia la portería" en jugadores de fútbol
}

\author{
Marcelo Correa Abuchacra ${ }^{1}$ \\ Fani Eta Korn Malerbi ${ }^{2}$
}

\section{Resumo}

O presente artigo teve por objetivo investigar os efeitos do reforçamento condicionado sobre a precisão do comportamento do "chute no gol" em jogadores de futebol em formação. A pesquisa foi realizada com dois participantes $e$ compreendeu duas fases: 1) Linha de Base (LB) e 2) Fase de Reforçamento. Foram colocados quatro alvos no gol (inferior direito, inferior esquerdo, superior direito e superior esquerdo) para indicar aos participantes onde deveriam chutar. Na LB apenas foram registrados os acertos ou erros dos "chutes no gol" de cada participante. Na Fase de Reforçamento cada acerto fazia com que o participante ganhasse um ponto que, ao final das sessões, era trocado por dinheiro. Para um dos participantes aumentou-se a quantidade de reforços para a precisão num dos alvos (Ref.+ bônus). Com o objetivo de verificar se a porcentagem de acertos retornaria aos níveis basais foi possível repetir o procedimento empregado na LB1, suspendendo o reforçamento condicionado aos acertos nos alvos IE e SE para o participante P1 e nos alvos SE, ID e SD

\footnotetext{
1 Psicólogo, especialista em Psicologia do Esporte e Mestre em Psicologia Experimental. E-mail: marcelo.abuchacra@gmail.com

2 Pontifícia Universidade Católica de São Paulo, SP, Brasil. E-mail: fanimalerbi@ terra.com.br
} 
para o participante $\mathrm{P} 2$. Os resultados mostraram que ambos os participantes elevaram seus índices de acerto na Fase de Reforçamento em comparação aos dados da Linha de Base.

Palavras-chave: reforçamento condicionado; alvos; chute no gol; precisão

\section{Abstract}

The aim of this article was to investigate the effects of conditioned reinforcement on the accuracy of the "kicking the ball into the goal" behavior in soccer players in training. The research was carried out with two participants and comprised two phases: 1)Base Line (LB) and 2) Reinforcement Phase. Four targets were placed on the goal (lower right, lower left, upper right and upper left) to indicate to the participants where they should kick. In LB only the hits or errors of the "kicking the ball into the goal" of each participant were recorded. In the Reinforcement Phase each hit scored one point that at the end of the sessions was exchanged for money. For one of the participants, the amount of reinforcement for the precision in one of the targets was increased (Ref. + Bonus). With the objective of verifying if the percentage of correct "kicks that made it into the goal" would return to Base Line levels it was possible to repeat the procedure used in $L B 1$ for the participant $P 1$ withdrawing the reinforcement to the hits in IE and SE targets and, for P2, to SE, ID and SD targets The results showed that both participants increased their hit rates in the Reinforcement Phase compared to the Base Line data.

Keywords: conditioned reinforcement; targets; kicking the ball into the goal; precision

\section{Resumen}

El presente artículo tuvo por objetivo investigar los efectos del refuerzo condicionado sobre la precisión del comportamiento del "tiro hacia la portería" en jugadores de fútbol en formación. La investigación fue realizada con dos participantes y comprendió dos fases: 1) Línea de Base (LB) y 2) Fase de Refuerzo. Se colocaron cuatro blancos (inferior derecha, inferior izquierda, superior derecha y superior izquierda) en la portería para indicar a los participantes donde debían patear. En la LB sólo se registraron los aciertos o errores de los "tiros en la portería" de cada participante. En la Fase de refuerzo, cada acierto hacía que el participante ganará un punto, que al final de las sesiones, era intercambiado por dinero. Para uno de los participantes se aumentó la cantidad de refuerzos para la precisión en uno de los blancos (Ref. + Bonificación). Con el objetivo de verificar si el porcentaje de aciertos retornaría a los niveles basales, fue posible repetir el procedimiento realizado en la LB1, suspendiendo el reforzamiento condicionado en los aciertos en los blancos Inferior izquierda y superior izquierda para el participante P1, y en los blancos superior izquierda, 
inferior derecha y superior derecha para el participante P2. Los resultados mostraron que ambos participantes aumentaron sus índices de acierto en la fase de reforzamiento en comparación a los dados de la Línea de Base.

Palabras clave: refuerzo condicionado; tiro en la portería; precisión

Entre todas as ações táticas executadas durante a partida de futebol, a cobrança de pênalti é, sem dúvida, um dos comportamentos decisivos para definir que time ganha o jogo.

Alguns estudos procuraram identificar quais são as variáveis que determinam os chutes corretos e verificaram que os chutes mais precisos são aqueles com menor velocidade (Timmis, Turner \& Van Paridon, 2014), os que ocorrem independentemente da possível reação do goleiro (Castillo, Oña, Raya, Bilbao \& Serra, 2010) e aqueles apresentados por jogadores que não tentaram enganar o goleiro no instante da cobrança (Smeeton \& Wiliams, 2012).

Estudos realizados para verificar a precisão dos chutes em alvos (inferiores e superiores), com o uso da captação das imagens dos chutes com câmeras de alta velocidade, tanto em condições estáticas como em movimento, identificaram diferenças na atividade muscular dos membros inferiores dos participantes (Finnoff, Newcomer \& Laskowski, 2002; Katis et al., 2013). Tais pesquisas verificaram que a atividade muscular foi associada à precisão dos chutes que, por mais que fossem velozes e potentes, não cumpriam o objetivo de acertar os alvos com a mesma eficácia que os chutes realizados com menor atividade muscular dos membros inferiores.

Alguns estudos observacionais usaram dispositivos e recursos tecnológicos para analisar o chute, enfocando variáveis como velocidade da bola (Markovic, Dizdar \& Jaric, 2006; Van den Tilaar \& Ulvik, 2014), os movimentos oculares dos jogadores (Giagazoglou, Katis, Kellis \& Natsikas, 2011; Peiyong \& Inomata, 2012), a potência e a posição do pé durante as repetições do movimento do chute (Shan \& Westerhoff, 2005; Inoue, Nunome, Sterzing, Shinkai \& Ikegami, 2014), tanto em condições estáticas, realizando os chutes com a bola parada (Wilson, Wood \& Vine 2009; Button, Dicks, Haines, Barker \& Davids, 2011), como em condições dinâmicas, 
executando o chute com a bola em movimento (Shinkai, Nunome, Isokawa \& Ikegami, 2009). Os dados tem apontado que as atividades musculares de outras partes do corpo (tronco, abdômen, ombros e braços), bem como os movimentos oculares do batedor, influenciam na velocidade e na potência do chute (Markovic, Dizdar \& Jaric, 2006; Van den Tilaar \& Ulvik, 2014; Giagazoglou et al.s, 2011). Os chutes precisos (aqueles que atingem o alvo) são, geralmente, mais lentos que os chutes imprecisos (Shan \& Westerhoff, 2005; Inoue et al., 2014; Wilson, Wood \& Vine, 2009; Button et al., 2011; Shinkai et al., 2009)

Navarro, Van der Kamp, Ranvaud e Savelsberg (2013) investigaram o papel das instruções na precisão das cobranças de penaltis por jogadores de futebol durante o treino. Um grupo de participantes foi instruído a selecionar o local do chute previamente e a ignorar os movimentos do goleiro e outro grupo não recebeu nenhuma instrução. Os resultados mostraram que o melhor desempenho foi do grupo que recebeu instruções.

A Análise do Comportamento tem acumulado evidências de que quando certos eventos ambientais seguem imediatamente alguma ação, essa ação torna-se mais provável de acontecer novamente no futuro (Skinner, 1938). O procedimento que fortalece a resposta dos indivíduos ao oferecer um estímulo como consequência da ocorrência daquela resposta é denominado reforçamento positivo e o evento que seguiu esta ação, reforço positivo.

A eficiência de um reforço varia dependendo da distância temporal entre a apresentação da resposta e a ocorrência do estímulo reforçador. Dessa forma, quanto menor o tempo de apresentação do estímulo reforçador após a resposta do indivíduo, maior será o seu valor reforçador. Alguns reforços positivos adquirem a propriedade de fortalecer as respostas que os precedem na história filogenética da sua espécie e são chamados de reforços primários (água, alimento, sexo e sono). Outros adquirem a função reforçadora no decorrer da vida do indivíduo e são chamados reforços secundários ou condicionados (Skinner, 1938).

Segundo Catania (1998), o reforçamento condicionado é o resultado de uma história de associação do estímulo inicialmente neutro com um 
estímulo reforçador já estabelecido. É importante ressaltar que a função reforçadora condicionada dos estímulos depende de manutenção do processo de condicionamento e de operações motivadoras.

Para Keller e Schoenfeld (1950) o reforçamento condicionado é um processo extremamente importante para atividades complexas em seres humanos. Os reforçadores condicionados são capazes de manter o comportamento na ausência de reforçadores primários. Dinheiro é um reforçador secundário generalizado, uma vez que pode ser trocado por coisas que sejam úteis ao indivíduo, no momento que necessitar, como por exemplo, comprar comida quando estiver com fome (Skinner, 1968). Pontos, fichas, troféus e medalhas também podem ser usados como reforçadores generalizados. Um aspecto relevante dos reforçadores generalizados é que eles não dependem de estados específicos de privação.

Thelen e Fisher (1983) investigaram os efeitos do reforçamento condicionado sobre as alterações topográficas do movimento e sobre as taxas de emissão de chutes espontâneos em crianças de três anos por meio de observações sistemáticas nos treinamentos. Verificaram que os participantes do grupo controle, que foram submetidos a reforços não contingentes ao comportamento de chutar, apresentaram menores taxas de emissão de chutes espontâneos que aqueles que tiveram os chutes reforçados imediatamente após a sua ocorrência, de forma sistemática, com elogios do treinador.

O objetivo do presente estudo foi verificar os efeitos de uma intervenção comportamental, que empregou reforçamento condicionado, sobre a precisão de chutes no gol, em alvos pré-determinados, em jogadores de futebol em formação.

\section{MÉTODO}

\section{Participantes}

Dois adolescentes, matriculados no Ensino Médio, que atuavam em um clube de futebol de São Paulo e que estavam inscritos na categoria sub 17 na Federação Paulista de Futebol representando o clube. Os atletas tinham 
sido contratados para jogar futebol pela instituição aos 14 anos de idade. A Tabela 1 apresenta as características dos participantes mostrando que ambos tinham o pé esquerdo como dominante e já haviam praticado futsal.

Tabela 1 - Características dos participantes

\begin{tabular}{ccccc}
\hline & IDADE & POSIÇÃo & PÉ DOMINANTE & $\begin{array}{c}\text { PRATICOU OUTRA } \\
\text { MODALIDADE }\end{array}$ \\
\hline P1 & 16 anos & Atacante & Esquerdo & Futsal \\
P2 & 16 anos & Meio campo & Esquerdo & Futsal \\
\hline
\end{tabular}

\section{Local}

A coleta de dados foi realizada no centro de treinamento do clube ao qual os participantes eram filiados.

\section{Material}

A meta regulamentar de futebol $(7,32 \times 2,44 \mathrm{~m})$ foi dividida em áreas de alvo superior e inferior, pendurando-se arcos de 75 centímetros de diâmetro, confeccionados em material flexível e fixados às traves com fita adesiva do tipo silver tape (Figura 1).

Foram utilizadas folhas de registro para a ocorrência de acertos e de erros em cada alvo.

\section{Procedimento}

A tarefa de chutar foi executada nos alvos Inferior Direito (ID), Inferior Esquerdo (IE), Superior Direito (SD) e Superior Esquerdo (SE) acoplados às traves do gol, sem a presença do goleiro. Foi demarcado um ponto fixo de chute a 11 metros (distância do pê- nalti) da meta para todos os participantes em todas as tentativas.

Cada sessão ocorria antes do treino habitual dos participantes no clube e envolvia cinco minutos de aquecimento e quatro séries de 16 chutes aos alvos. 


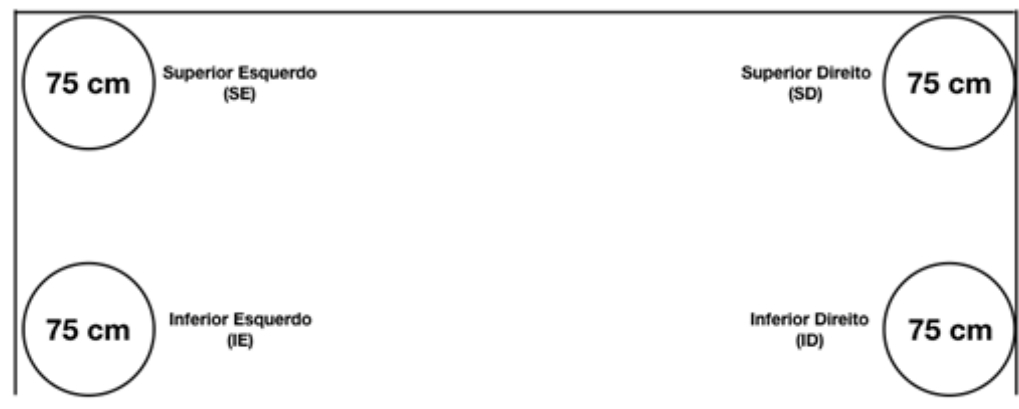

$11 \mathrm{~m}$

\section{Ponto de Chute}

Figura 1 - Disposição e medidas dos quatro alvos dentro do gol e da distância padrão do ponto de chute.

Aquecimento. Cada participante foi orientado a realizar um aquecimento durante cinco minutos, nos membros inferiores, para ativação da musculatura necessária para os chutes. O pesquisador dizia para os participantes: "Antes de iniciar o procedimento, você deverá aquecer seu corpo, principalmente os membros inferiores, durante cinco minutos. Realize o aquecimento da forma como preferir, mas lembre-se que um bom aquecimento pode ajudá-lo a iniciar bem os treinos de chutes aos alvos".

Linha de Base $(L B)$ 1. Com o objetivo de avaliar a precisão dos chutes ao alvo sem reforçamento, após o aquecimento os participantes foram solicitados a executar quatro chutes consecutivos em cada um dos alvos. A ordem da escolha dos alvos foi estabelecida pelos próprios participantes. Nesta fase, o pesquisador dizia aos participantes: “Agora será dado o início ao treino de chutes nos alvos. Você irá executar 64 chutes por sessão com o seu pé dominante. É necessário executar quatro chutes consecutivos num determinado alvo para que possa passar para os próximos alvos. Após 
iniciar os chutes, você deve, obrigatoriamente, executar quatro chutes no alvo escolhido, para assim, ter acesso ao alvo seguinte, até que os 16 chutes (quatro em cada alvo) sejam apresentados. Após os primeiros 16 chutes, você terá um tempo de descanso (três a cinco minutos) enquanto um outro participante entrará no seu lugar. Assim que a série do outro participante terminar, você iniciará sua segunda série de 16 chutes, repetindo a ordem de escolha dos alvos da primeira série. Caso sinta algum desconforto durante o treino, pode interrompê-lo no momento que julgar necessário.”.

Os chutes que faziam a bola bater na área do alvo ou passavam por dentro das esferas foram considerados acertos. Na LB, o primeiro autor apenas comentava que havia ocorrido acerto ou erro. A LB terminava para um determinado alvo para um determinado participante após a porcentagem de acertos atingir uma estabilidade, isto é, ocorrer com uma variação até $10 \%$ em três sessões consecutivas.

Cada alvo foi considerado independentemente, ou seja, se ocorresse uma estabilidade na porcentagem de acertos para aquele alvo, ocorria mudança de fase apenas para aquele alvo.

Reforçamento. Nessa fase, após o aquecimento, os atletas foram informados que receberiam pontos pelos acertos (1 ponto por acerto), os quais poderiam ser trocados por dinheiro ao final das sessões. O primeiro autor fornecia as seguintes instruções: "A partir desta sessão, uma nova regra será inserida nos seus treinos de chutes nos alvos. A cada chute que acertar no alvo X somará pontos, os quais poderão ser trocados por dinheiro no final da sessão, cada acerto valendo $\mathrm{R} \$ 1, \mathrm{oo}$. Nos demais alvos, cada acerto ou erro serão apenas registrados por mim. Caso sinta algum desconforto durante o treino, pode interrompê-lo no momento que julgar necessário".

Reforçamento + bônus. Para o participante P2, que precisou de 37 sessões para atingir a estabilidade nos acertos no alvo inferior esquerdo (IE) durante a LB1, foi acrescentado um bônus para os acertos neste alvo. A instrução foi: "Neste alvo (IE) cada acerto continua valendo R \$1,00 mas, a cada quatro chutes consecutivos que você acertar, você receberá um bônus de R\$2,00”. 
Linha de Base 2. Com o objetivo de verificar se o aumento na porcentagem de acertos foi decorrente do reforçamento ou constituiu um efeito da prática, repetiu-se o procedimento empregado na LB1, suspendendo o reforçamento condicionado aos acertos nos alvos IE e SE para o participante P1 e nos alvos SE, ID e SD para o participante P2. A suspensão do reforçamento condicionado ocorreu com base no mesmo critério de estabilidade - variação maxima de 10\% na porcentagem de acertos - em três sessões consecutivas da Fase de Reforçamento.

\section{RESULTADOS}

A Figura 2 apresenta a porcentagem de acertos obtidos pelo Participante 1 (P1) nos quatro alvos em cada sessão de cada fase experimental: Linha de Base 1 (LB1), Reforçamento (RC) e Linha de Base 2 (LB2).

Como se pode ver na Figura 2, a duração da Fase Linha de Base 1 variou para os diferentes alvos para o participante P1 porque a estabilidade na porcentagem de acertos foi atingida após um número diferente de sessões para cada alvo. No início da Fase de Reforçamento, ocorreu uma grande variabilidade na porcentagem de acertos principalmente para o alvo superior direito, indicando a ocorrência de uma fase de transição entre dois estados estáveis. Para os alvos IE e SE, para os quais foi possível repetir o procedimento da Linha de Base (LB2), ocorreu um novo estado de transição antes de a porcentagem de acertos atingir uma outra estabilidade.

A Tabela 2 apresenta as médias e os desvios-padrões das porcentagens de acertos apresentados por P1, para cada alvo, em cada fase do estudo. Como se pode ver, na LB1 o participante P1 apresentou diferentes índices de acertos para os diferentes alvos, com valores maiores nos alvos inferiores, sugerindo que estes eram mais fáceis para este participante. Com a introdução do reforçamento, houve um aumento na média da porcentagem de acertos para todos os alvos. A suspensão do reforçamento para os alvos IE e SE foi acompanhada de uma diminuição nos índices médios de acertos, sem retornarem, no entanto, aos níveis basais. 


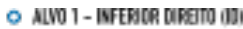
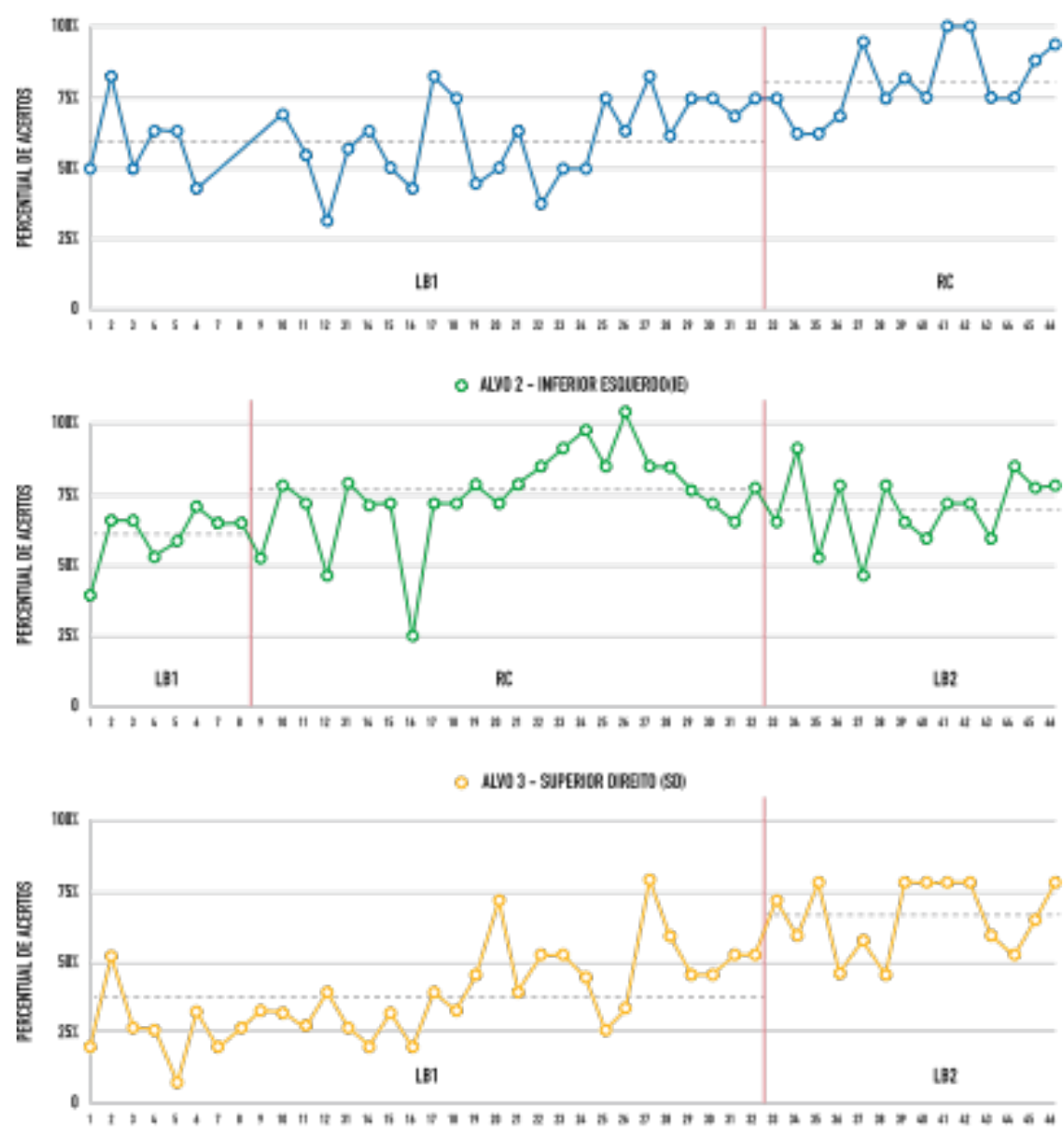

O ALV 6 - SUPEALR ESOUEATO ISE

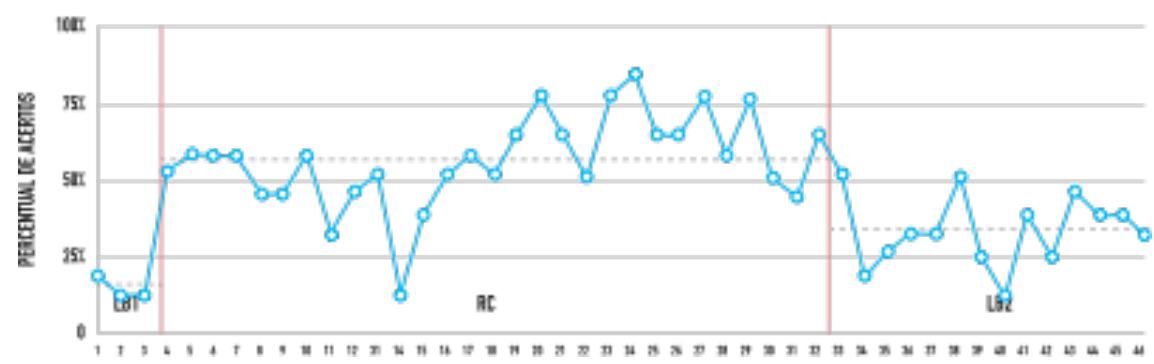

Figura 2 - Porcentagem de acertos de P1 nos alvos ID, IE, SD e SE nas fases LB1, Reforçamento e LB2 (alvos IE e SE) e respectivas médias de cada fase representadas pela linha pontilhada. 
Tabela 2 - Médias e desvios-padrões das porcentagens de acertos de P1 em cada alvo, na ordem de preferência de escolha do participante, nas diferentes fases

\begin{tabular}{lcccc}
\hline & $\mathrm{ID}$ & $\mathrm{IE}$ & $\mathrm{SD}$ & $\mathrm{SE}$ \\
\hline \multirow{2}{*}{ Linha de Base 1 } & $59,77 \%$ & $57,81 \%$ & $36,13 \%$ & $14,58 \%$ \\
& $(\mathrm{DP}=2,13 \%)$ & $(\mathrm{DP}=2,48 \%)$ & $(\mathrm{DP}=2,43 \%)$ & $(\mathrm{DP}=0,57 \%)$ \\
Reforçamento & $80,36 \%$ & $71,61 \%$ & $63,39 \%$ & $54,74 \%$ \\
& $(\mathrm{DP}=2,03 \%)$ & $(\mathrm{DP}=2,48 \%)$ & $(\mathrm{DP}=1,95 \%)$ & $(\mathrm{DP}=2,32 \%)$ \\
Linha de Base 2 & - & $66,96 \%$ & - & $32,59 \%$ \\
& - & $(\mathrm{DP}=1,97 \%)$ & - & $(\mathrm{DP}=1,76 \%)$ \\
\hline
\end{tabular}

Aplicou-se o teste t pareado para comparar as médias das porcentagens de acertos nos quarto alvos, nas fases Linha de Base 1 e Reforçamento, uma vez que não se obteve dados da LB2 para todos os alvos. Essa análise estatística mostrou ter ocorrido uma diferença significativa $(p=0,02)$ na porcentagem de acertos na fase de Reforçamento em relação à LB1.

A Figura 3 apresenta a porcentagem de acertos obtida pelo Participante 2 ( $\mathrm{P} 2)$ nos quatro alvos em todas as fases experimentais: Linha de Base 1 (LB1), Reforçamento (RC), Reforçamento + bônus (Ref. + bônus) e Linha de Base 2 (LB2).

Como se pode ver na Figura 3, a estabilidade na porcentagem de acertos no alvo ID foi atingida em apenas quarto sessões na LB1, mas precisou de 33 sessões na Fase de Reforçamento para ser alcançada. No alvo SD, a LB1 foi mais longa (29 sessões) e a Fase de Reforçamento mais curta (apenas sete sessões). Quando se aumentou a magnitude do reforço (condição de reforçamento com bônus) para a precisão no alvo IE, P2 apresentou índices de acertos superiores àqueles da LB1 em todas as sessões dessa fase. Com a suspensão do reforçamento (LB2) nos alvos ID, SE e SD foi possível observar uma queda na média dos índices de acertos em comparação com a Fase de Reforçamento em todos os alvos. 

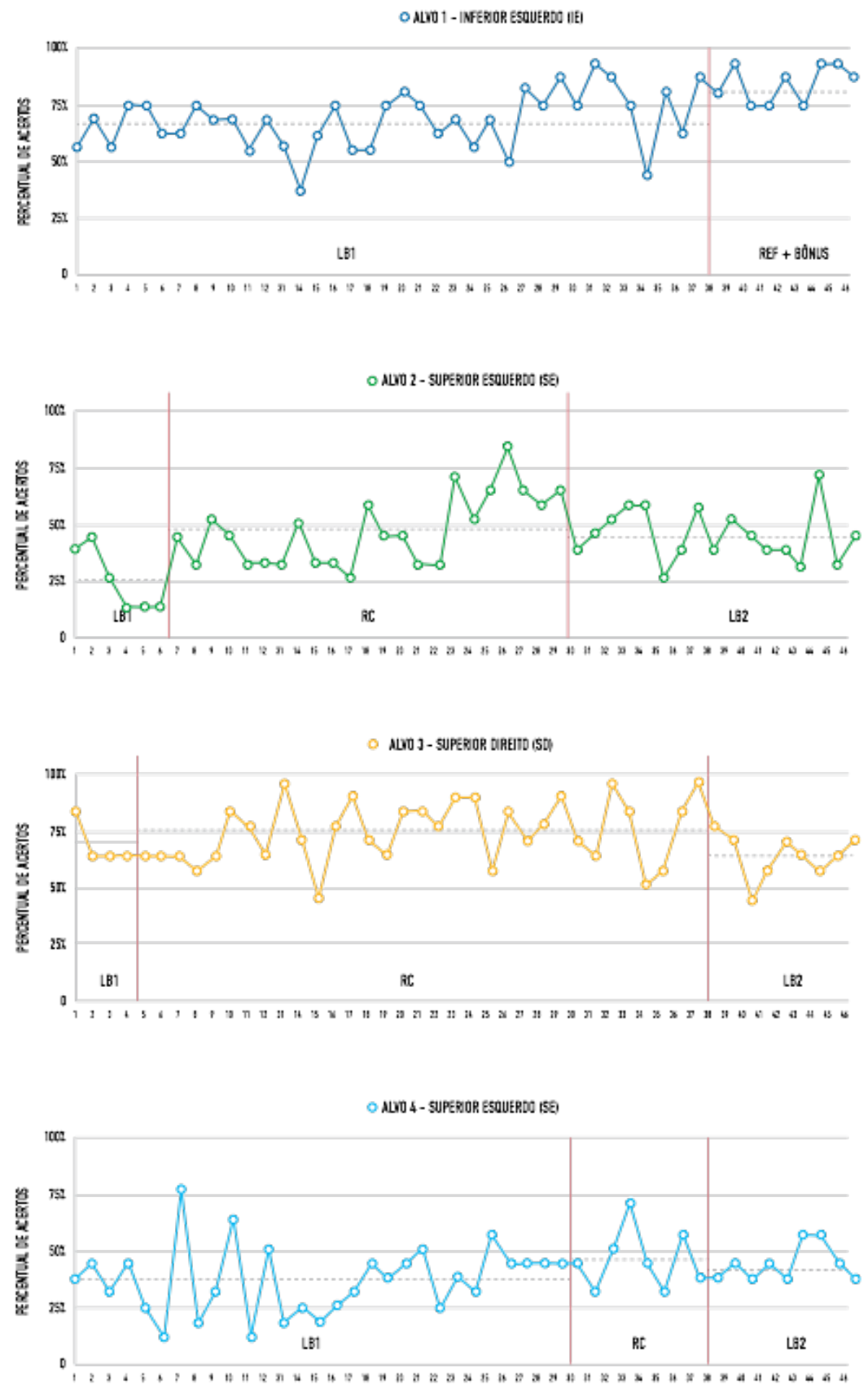

Figura 3 - Porcentagem de acertos de P2 nos alvos IE, SE, ID e SD nas fases LB1, Reforçamento e LB2 (alvos SE, ID e SD) e respectivas médias de cada fase representadas pela linha pontilhada. 
A Tabela 3 apresenta as médias e os desvios-padrões das porcentagens de acertos apresentados por P2 em cada fase para cada alvo. Como se pode ver, na LB1 o participante P2 também apresentou diferentes índices de acertos para os diferentes alvos, sugerindo que para este participante também os alvos inferiores eram mais fáceis do que os superiores.

Replicando os dados obtidos com o participante P1, a introdução do reforçamento foi acompanhada por um aumento na média da porcentagem de acertos para todos os alvos. Novamente, com a suspensão do reforçamento para os alvos SE, ID e SD, houve uma diminuição nos índices médios de acertos sem retornarem, no entanto, aos níveis basais.

Tabela 3 - Médias e desvios-padrões das porcentagens de acertos de P2 em cada alvo, na ordem de preferência de escolha do participante, nas diferentes fases

\begin{tabular}{lcccc}
\hline & $\mathrm{IE}$ & $\mathrm{SE}$ & $\mathrm{ID}$ & $\mathrm{SD}$ \\
\hline \multirow{2}{*}{ Linha de Base 1} & $68,24 \%$ & $23,96 \%$ & $67,19 \%$ & $36,64 \%$ \\
& $(\mathrm{DP}=2,01 \%)$ & $(\mathrm{DP}=2,22 \%)$ & $(\mathrm{DP}=1,5 \%)$ & $(\mathrm{DP}=2,35 \%)$ \\
Reforçamento & $84,72 \%$ & $45,65 \%$ & $72,54 \%$ & $45,31 \%$ \\
& $(\mathrm{DP}=1,33 \%)$ & $(\mathrm{DP}=2,42 \%)$ & $(\mathrm{DP}=2,12 \%)$ & $(\mathrm{DP}=2,05 \%)$ \\
Linha de Base 2 & - & $43,75 \%$ & $62,50 \%$ & $43,75 \%$ \\
& - & $(\mathrm{DP}=1,8 \%)$ & $(\mathrm{DP}=1,5 \%)$ & $(\mathrm{DP}=1,22 \%)$ \\
\hline
\end{tabular}

A aplicação do teste t pareado para comparar as médias das porcentagens de acertos, nos quarto alvos, nas fases Linha de Base 1 e Reforçamento, mostrou ter ocorrido uma diferença significativa $(\mathrm{p}=0,039)$ nos índices de acertos na fase de Reforçamento em relação à LB1 também para o participante P2.

\section{DISCUSSÃO}

A análise da precisão dos chutes aos diferentes alvos na fase LB1 (antes que se oferecesse reforçamento para os acertos) sugeriu que cada alvo apresentava um nível de dificuldade diferente para os participantes do estudo. Para ambos os participantes, os alvos superiores parecem ter se mostrado mais difíceis do que os inferiores. Tanto para P1 como para P2, 
os alvos SD e SE foram associados com os menores índices de acertos na fase LB1. Coincidentemente, na fase de reforçamento, foi no alvo superior esquerdo que ocorreu o maior aumento do índice de precisão em comparação com os demais alvos para ambos os participantes.

Independentemente da dificuldade associada com cada alvo, foi possível observar que o reforçamento das respostas corretas aumentou a precisão dos chutes para ambos os participantes do presente estudo.

O aumento da precisão dos chutes pôde ser mais claramente observado nas situações nas quais os índices de acertos na LB1 eram baixos, a saber, no alvo SE para ambos os participantes. Foi possível observar que o critério de estabilidade na LB1 foi atingido no alvo SE em seis sessões para P2 e em três sessões para P1 com baixos índices de acerto, possibilitando uma variação maior na precisão dos chutes a este alvo na fase de Reforçamento.

É importante ressaltar que para P2, o procedimento de Reforçamento no alvo IE foi diferente daquele aplicado no outro participante, uma vez que além da atribuição de pontos por cada acerto, o pesquisador ofereceu um bônus adicional, caso o participante acertasse os quatro chutes da série, aumentando assim a proporção de reforços por acertos. Verificou-se que, nesta condição de intervenção com bônus, P2 apresentou todos os índices de precisão superiores à média obtida na LB1, bem como a conquista de um novo pico máximo de desempenho em três das nove sessões realizadas na condição com bônus.

A comparação do desempenho dos participantes nos alvos que foram submetidos à remoção do Reforçamento (LB2) com o desempenho nas fases anteriores indicou que, na maioria dos casos, a porcentagem de acertos não voltou aos níveis da LB1, o que sugere que a precisão dos chutes deve ter ficado sob o controle de reforços diferentes dos pontos que eram trocados por dinheiro. Robinson (1998) discute algumas questões relacionadas ao uso do reforçamento em programas de promoção à saúde, admitindo que apesar do efeito positivo do esquema de reforçamento arbitrário e da sua funcionalidade na aquisição de habilidades, não é dada a devida atenção às 
consequências naturais que podem passar a controlar o comportamento, que é o que se espera que aconteça na situação real fora do ambiente de pesquisa.

Num estudo realizado com crianças, Thelen e Fisher (1983) observaram resultados positivos em relação à aplicação do reforço condicionado imediato (elogios do treinador) após a ocorrência dos chutes nos participantes do grupo experimental em relação ao grupo controle. Alvarez, Balaguer, Castillo e Duda (2009) e Garcia-Mas et al. (2010) mostraram que os elogios fornecidos como consequência das ações técnicas dos jogadores de futebol como chutes apropriados, passes corretos, seguimento de instruções, etc. são variáveis que controlam também o prazer de praticar e competir em atletas em formação. É possível considerar que, no presente estudo, a atenção social fornecida pelo pesquisador após os acertos dos chutes tenha também contribuído para a manutenção da precisão dos chutes, mesmo após a suspensão do reforçamento por pontos/dinheiro.

$\mathrm{Na}$ presente pesquisa cada participante apresentava no início do estudo um repertório técnico (e. g. padrões do movimento de chutar em cada alvo, forma de executar o contato do pé com a bola, inclinação do corpo, ângulo do joelho, quantidade de força, etc) provavelmente aprendido ao longo de sua história no futebol, modelado de acordo com as consequências produzidas no ambiente. Geralmente, o treinamento de bolas paradas (escanteios, faltas e pênaltis), tanto nas categorias de base como em equipes profissionais, é realizado através de repetições e da produção da consequência do acerto, ou seja, o gol. Com base nos acertos ou erros emitidos pelos jogadores no treinamento, o treinador determina quem serão os atletas responsáveis por realizar as respectivas cobranças de bolas paradas (Cunha, 2007). Os resultados obtidos pela presente pesquisa fornecem pistas para aprimorar o treinamento dos jogadores em situações de bola parada, como por exemplo, nas cobranças de pênaltis, sugerindo possibilidades reais e mensuráveis de aumento da precisão do chute ao gol.

A presente pesquisa enfocou o comportamento de chute no gol numa situação que simulou cobranças de pênaltis. Considerando que acertar a meta é a consequência mais valiosa da modalidade, podemos refletir sobre a possibilidade de aplicar o procedimento de reforçamento condicionado 
tanto para categorias de base como em equipes profissionais. Associar o acerto em determinado alvo a um estímulo condicionado de alto valor reforçador pode produzir maior motivação nos treinamentos o que contribuiria para aprimorar o desempenho dos atletas.

Entretanto, a variabilidade na porcentagem de acertos observada em cada fase, em cada alvo, para cada participante do presente estudo, sugere que variáveis estranhas não identificadas podem também ter controlado a precisão dos chutes. Pesquisas posteriores deveriam identificar e controlar tais variáveis.

\section{REFERÊNCIAS}

Alvarez, M. S., Balaguer, I., Castillos, I., \& Duda, J. L. (2009). Coach autonomy support and quality of sport engagement in young soccer players. The Spanish Journal of Psychology, 12 (1), 138-148.

Button, C., Dicks, M., Haines, R., Barker, R., \& Davids, K. (2011). Statistical modelling of gaze behaviour as categorical time series: what you should watch to save soccer penalties. International Quarterly of Cognitive Science, 12 (3), 235-244.

Castillo, J. M., Oña, A., Raya, A., Bilbao, A. \&, Serra, E. (2010). Tactical skills and ball speed during a field simulation of penalty kick strategies in soccer. Percept Mot Skills, 111 (3), 947-962.

Catania, C. A. (1998). Learning. 4 ed. New Jersey: Prentice Hall.

Cunha, N. (2007). A importância dos lances de bola parada (livres, cantos e penaltis) no Futebol de 11. Análise de situações finalizadas com golo na 1 a Liga Portuguesa 2005/o6 e no Campeonato do Mundo 2006. (Dissertação de Licenciatura). Faculdade de Desporto, Universidade do Porto, Porto.

Finnoff, J. T., Newcomer, K. \& Laskowski, E. R. (2002). A valid and reliable method for measuring the kicking accuracy of soccer players. The Journal of Science and Medicine in Sport, 5 (4), 348-353. 
Garcia-Mas, A., Palou, P., Gili, M., Ponseti, X., Borras, P. A., Vidal, J., ... Sousa, C. (2010). Commitment, enjoyment and motivation in young soccer competitive players. The Spanish Journal of Psychology, 13 (2), 609-616.

Giagazoglou, P., Katis, A., Kellis, E., \& Natsikas, C. (2011). Differences in soccer kick kinematics between blind players and controls. Adapted Physical Activity Quarterly, 28 (3), 251-266.

Inoue, K., Nunome, H., Sterzing, T., Shinkai, H. \& Ikegami, Y. (2014). Dynamics of the support leg in soccer instep kicking. The Journal of Sports Science and Medicine, 32 (11), 1023-1032.

Katis, A., \& Kellis, E. (2011). Is soccer kick performance better after a "faking" (cutting) maneuver task? Sports Biomechanics, 10 (1), 35-45.

Katis, A., Giannadakis, E., Kannas, T., Amiridis, I., Kellis, E., \& Lees, A. (2013). Mechanisms that influence accuracy of the soccer kick. Journal Electromyogr Kinesiology, 23 (1), 125-131.

Keller, F. S., \& Schoenfeld, W. N. (1950). Principles of psychology. New York: Appleton- Century-Crofts.

Markovic, G., Dizdar, D., \& Jaric, S. (2006). Evaluation of tests of maximum kicking performance. The Journal of Sports Medicine and Physical Fitness, 46 (2), 215-220.

Navarro, M., Van der Kamp, J., Ranvaud, R., \& Savelsbergh, G. J. (2013). The mere presence of a goalkeeper affects the accuracy of penalty kicks. Journal of Sports Sciences, 31 (9), 921-929.

Peiyong, Z., \& Inomata, K. (2012). Cognitive strategies for goalkeeper responding to soccer penalty kick. Perceptual and Motor Skills, 115 (3), 969-983.

Robison, J. I. (1998). To reward?... Or not to reward?: Quetioning the wisdom of using ex- ternal reinforcement in Health Promotion Programs. American Journal of Health Promoti- on, 13 (1), 1- 3.

Shan, G., \& Westerhoff, P. (2005). Full-body kinematic characteristics of the maximal instep soccer kick by male soccer players and parameters related to kick quality. Sports Biomechanic, 4 (1), 59-72. 
Shinkai, H. Nunome, H. Isokawa, M., \& Ikegami, Y. (2009). Ball impact dynamics of instep soccer kicking. Medicine Science Sports Exercise, 41 (4), 889-897.

Smeeton, N. J., \& Williams, A. M. (2012). The role of movement exaggeration in the anticipation of deceptive soccer penalty kicks. British Journal of Social Psychology, 174, 97-108.

Skinner, B. F. (1938). The behavior of organisms. New York: Appleton-Century-Crofts.

Skinner, B. F. (1968) The Technology of Teaching. New York: Appleton-Century-Crofts.

Thelen, E., \& Fisher, D. M. (1983). From spontaneous to instrumental behavior: kinematic analysis of movement changes during very early learning. Child Mental Health Development, 54 (1), 129-140.

Timmis, A. M., Turner, K., \& Van Paridon, N. K. (2014). Visual search strategies of soccer players executing a power vs. placement penalty kick. Sport and Exercise Sciences Research Group, 9 (12), 1-16.

Van Den Tillaar, R., \& Ulvik, A. (2014). Influence of instruction on velocity and accuracy in soccer kicking of experienced soccer players. Journal Motor Behavior, 46 (5), 287-291.

Wilson, M. R., Wood, G., \& Vine, S. J. (2009). Anxiety, attentional control, and performance impairment in penalty kicks. The Journal of Sport \& Exercise Psychology, 31 (6), 731-775. 